\title{
Perfil epidemiológico da hipospádia na Região Bragantina do estado de São Paulo, Brasil
}

\author{
Epidemiological profile of hypospadias in the Bragantina region of the state of \\ São Paulo, Brazil
}

Marco Aurélio Veiga Conrado ${ }^{1}$ (D), André Luiz Veiga Conrado² (D), Cibele Silva ${ }^{1}$ (D), Daniela Silvestre ${ }^{3}$ (D), Pedro Izzo 4 (D)

\begin{abstract}
RESUMO
Modelo de estudo: Estudo de prevalência. Objetivo do estudo: Avaliar o perfil epidemiológico dos casos de hipospádia atendidos no Hospital São Francisco na Providência de Deus da Universidade São Francisco (HUSF), Bragança Paulista/SP nos últimos 10 anos (2007-2017), relacionando os tipos de hipospádia com as malformações associadas e idade à primeira cirurgia corretiva. Metodologia: Foi realizado um levantamento de casos de hipospádia atendidos entre 2007 e 2017 no HUSF, com pacientes oriundos das cidades da Região Bragantina do estado de São Paulo. Os tipos de hipospádia foram relacionados com as malformações associadas. Foram pesquisados nos prontuários: tipo de hipospádia, idade à primeira cirurgia, laudo de exame ultrassonográfico, etnia, local de nascimento e malformação associada. Os dados foram analisados estatisticamente utilizando os testes Qui-quadrado, teste F e de Kruskal-Wallis. Resultados: Em 1401 atendimentos pediátricos no período avaliado, foram encontrados 45 casos de hipospádia com prevalência igual a 0,7/1000 nascidos vivos. Do total de casos, 29 foram classificados como do tipo distal, seis casos de hipospádia média e 10 casos do tipo proximal [F-test $\left.(2,8)=5,552 ; P=0,003 ; X^{2}=9,3117 ; P=0,009\right]$. Nos casos de hipospádia distal, foram detectadas 16 malformações associadas e, nos tipos média e proximal, foram observadas sete malformações associadas em cada uma $(K W=3,322, \mathrm{P}=0,19)$. O maior número de casos foi abordado cirurgicamente no intervalo entre os três e quatro anos de idade. Conclusão: A hipospádia distal foi a mais prevalente, com idade à primeira cirurgia em desacordo com o intervalo proposto para a correção da hipospádia.
\end{abstract}

Palavras-chave: Hipospádia; Malformações; Bragança Paulista.

\begin{abstract}
Study model: a Prevalence study. Objective: Evaluating the epidemiological profile of hypospadias cases assisted in the Hospital São Francisco na Providência de Deus of the Universidade São Francisco (HUSF), Bragança Paulista, Brazil, in the last 10 years (2007-2017), relating the types of hypospadias with associated malformations and age at the first corrective surgery. Methodology: A survey of hypospadias cases assisted between 2007 and 2017 in HUSF was carried out with patients from the Bragantina region of the state of São Paulo. The types of hypospadias were related to the associated malformations. The following data were collected from the medical records: type of hypospadias, age at first surgery, ultrasound imaging, ethnicity, place of birth, associated malformation. Data were statistically analyzed using Chi-squared, F-test and Kruskal-Wallis tests. Results: In 1401 pediatric assistances, it was found 45 cases of hypospadias with a prevalence of $0.7 / 1000$ births. From the total, 29 cases were classified as distal, six cases of medium, and 10 cases as proximal types [F-test $(2.8)=5.552, \mathrm{P}=0.003, \mathrm{X} 2=9.3117, \mathrm{P}=0.009$ ]. Cases of distal hypospadias had 16 associated malformations, and for medial and proximal types there were seven associated malformations in each one $(\mathrm{KW}=3.322, \mathrm{P}=0.19)$. The largest number of cases was surgically corrected between three and four years of age. Conclusion: Distal hypospadias were the most prevalent in the Bragantina region in the last 10 years. It was also observed that the age at first surgery is in disagreement with the range proposed for the corrective approach of hypospadias.
\end{abstract}

Keywords: Hypospadias; Malformations; Bragança Paulista.

1. Médico residente em cirurgia geral. Hospital São Francisco na Providência de Deus, Universidade São Francisco (HUSF), Bragança Paulista (SP), Brasil.

2. Médico veterinário, doutorando. Instituto de Ciências Biomédicas, Universidade de São Paulo (ICB/USP), São Paulo (SP), Brasil.

3. Cirurgiã pediátrica. HUSF, Bragança Paulista (SP), Brasil.

4. Mestre em cirurgia pediátrica. HUSF, Bragança Paulista (SP), Brasil.

$\triangle$ André Luiz Veiga Conrado. Laboratório de Histofisiologia Evolutiva - ICB-1, sala 409. Av. Prof. Lineu Prestes, 1524 - Cidade Universitária. CEP: 05508-000. São Paulo (SP), Brasil. andreveigaconrado@gmail.com | Recebido: 13/11/2018 | Aprovado: 03/04/2019 


\section{INTRODUÇÃO}

C onsidera-se a hipospádia uma malformação congênita clinicamente caracterizada pelo desenvolvimento incompleto da uretra com disposição do meato uretral na face inferior do pênis (face ventral) e não na extremidade da glande. A palavra origina-se do grego hypos (abaixo) e spadon (rasgado). Não é uma malformação exclusiva do sexo masculino, pois, também há hipospádia feminina caracterizada por abertura anormalmente alta da uretra no introito vaginal e, geralmente, acompanhada de certo grau de incontinência urinária ${ }^{1}$.

O desenvolvimento do sistema urinário apresenta íntima relação com o sistema reprodutor e a genitália externa. Apenas a partir da sexta semana de gestação é que se inicia a diferenciação do tubérculo genital e das pregas genitais em direção ao aparelho reprodutor masculino ou feminino, de acordo com a presença ou não de hormônio sexual masculino. Sabe-se que a diferenciação do trato genitourinário para o fenótipo masculino exige três hormônios: testosterona, 5-alfa diidrotestosterona, e a substância inibidora de Müller. Essa última induz a regressão do ducto de Müller; a testosterona estimula a diferenciação das estruturas derivadas do ducto de Wolff (epidídimo, vaso deferente e vesícula seminal) e a 5-alfa diidrotestosterona atua na diferenciação do seio urogenital. A base etiopatogênica da hipospádia é uma deficiência de testosterona durante a fase crítica de morfogênese da uretra (da nona à décima terceira semana de gestação) ${ }^{2}$.

Na maior parte das vezes, o quadro é acompanhado por curvatura peniana ventral congênita que se manifesta em decorrência de tecido fibroso ventral (resquício do corpo esponjoso hipoplásico) ou desproporção dos corpos cavernosos. Também pode ser percebido pelos pais pela presença de prepúcio exuberante e redundante apenas na face dorsal do pênis ${ }^{3}$. Entretanto, o diagnóstico é realizado ao nascimento pelo exame físico, na maioria dos casos $^{1}$.

De acordo com a posição do meato uretral, a hipospádia é classificada como distal ( $70 \%$ dos casos - glandular, coronal e peniana anterior); média ( $10 \%$ dos casos - peniana média) e proximal ( $20 \%$ dos casos - penoscrotal, escrotal e perineal) ${ }^{4}$.
É comum observar outras anomalias do trato urogenital associadas com a hipospádia, tais como, alterações do descenso testicular em 9,3\%, chegando até $31,6 \%$ nas formas proximais (escrotal e penoscrotal), além de distúrbios do desenvolvimento sexual ${ }^{5}$. Ikoma et al. (1985) ${ }^{6}$ observaram $27,5 \%$ de cisto de utrículo em 280 pacientes com hipospádia investigados radiologicamente. Pode ser encontrada associada à ectopia renal, hérnia inguinal bilateral, além de anomalias não associadas com o trato urinário, como a estenose aórtica?.

O tratamento da malformação é cirúrgico e visa a correção estética e funcional da genitália masculina. O prognóstico geralmente é bom e o sucesso da cirurgia está relacionado diretamente com a familiaridade do profissional com a técnica empregada ${ }^{8}$.

Algumas publicações têm demonstrado que a prevalência da hipospádia praticamente dobrou a partir da década de $70^{9}$, porém, em prematuros, esse número atinge uma frequência 10 vezes maior $^{10}$. Esse crescimento reforça a necessidade de um estudo epidemiológico pormenorizado dos casos dessa malformação.

Diante do exposto, esta pesquisa teve como objetivo avaliar a prevalência dos casos de hipospádia atendidos no Hospital Universitário São Francisco na Providência de Deus de Bragança Paulista/SP, de 2007 a 2017, relacionando os tipos de hipospádia e as malformações associadas.

\section{MATERIAL E MÉTODOS}

Foi realizado um levantamento de casos de hipospádia atendidos entre 2007 e 2017 no Hospital São Francisco na Providência de Deus da Universidade São Francisco (HUSF), Bragança Paulista/SP, oriundos das cidades que compõem a Região Bragantina do estado de São Paulo (Figura 1). Neste estudo retrospectivo, os tipos de hipospádia foram relacionados com as malformações associadas. Foram pesquisados os seguintes dados nos prontuários: tipo de hipospádia, idade em que se realizou a primeira cirurgia, laudo de exame ultrassonográfico, etnia, local de nascimento e malformação associada.

A prevalência da hipospádia foi calculada considerando o número total de nascidos vivos 
de ambos os sexos na Região Bragantina, recuperados do Sistema de Informação Hospitalar do DATASUS (SIH/SUS) no período estudado. Deve-se se salientar que o HUSF é o hospital de referência da Região Bragantina para intervenções cirúrgicas pediátricas.

Os tipos de hipospádia, número de casos e as alterações foram analisados estatisticamente utili- zando o teste de Qui-quadrado $\left(\mathrm{X}^{2}\right)$, teste $\mathrm{F}$ (F-test) e Kruskal-Wallis (KW) com intervalo de confiança de $95 \%$ utilizando-se o programa estatístico GraphPad Prism 6 (GraphPad Software, Inc, USA).

Esta pesquisa foi aprovada pelo Comitê de Ética em Pesquisa do Hospital São Francisco na Providência de Deus, Plataforma Brasil n. 0 2.684.581.
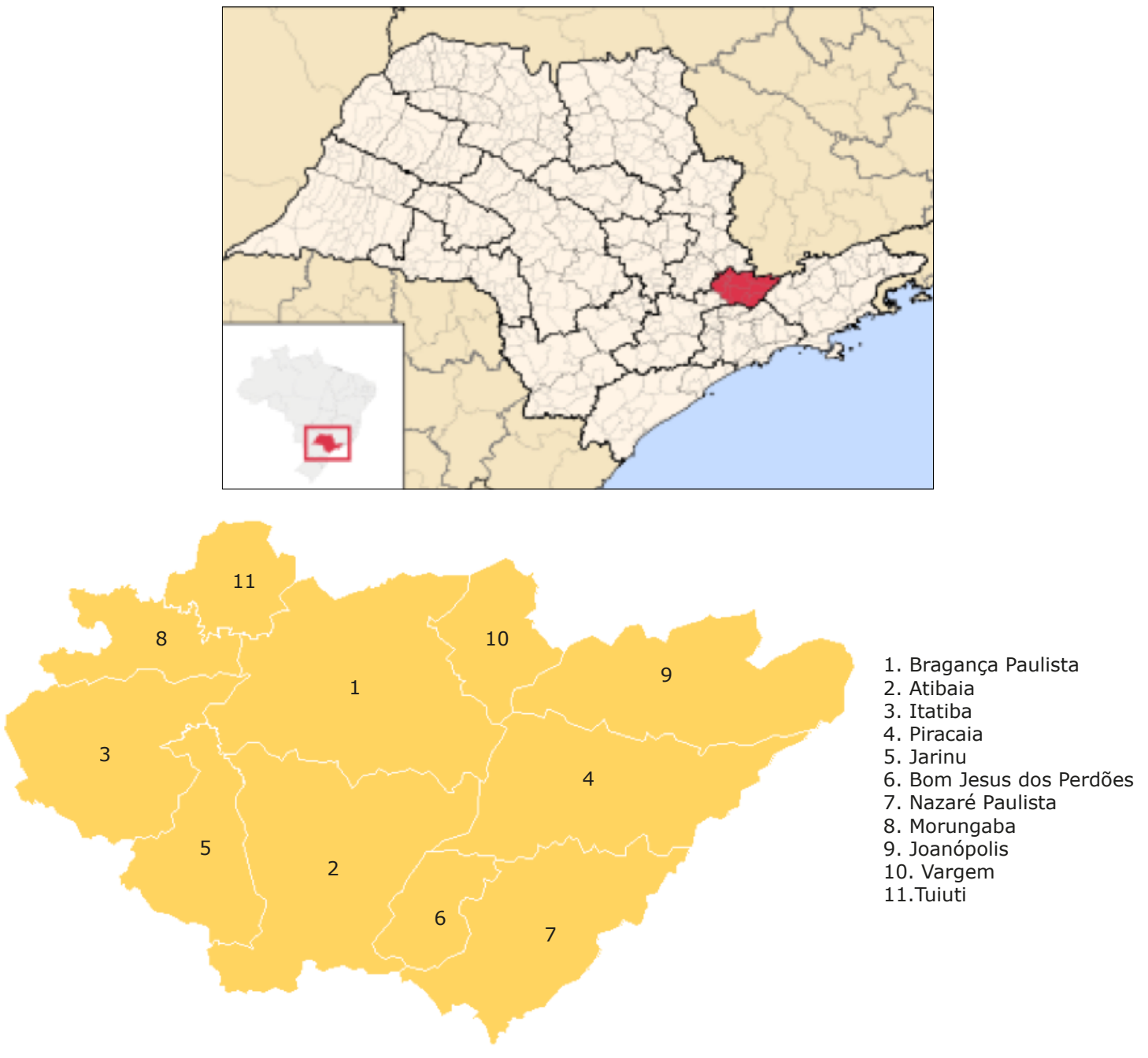

Figura 1: Localização da microrregião de Bragança Paulista no estado de São Paulo e seus municípios integrantes. (Fonte: Wikipedia).

\section{RESULTADOS}

A partir do acesso ao SIH/SUS, encontrou-se o registro de 1401 atendimentos pediátricos (clínicos e cirúrgicos) no período entre 2007 e 2017. Des- tes, 45 foram correções de hipospádia realizadas no HUSF, representando $3,2 \%$ dos procedimentos do período estudado. Entre 2007 e 2017, o número de nascidos vivos foi de 63.681 na Região Bragantina, com prevalência de hipospádia de 0,7/1000 nas- 
cidos vivos. Para este cálculo foi excluído um caso em que o paciente era oriundo de Monte Verde/MG.

No presente estudo, com relação à localização do meato hipospádico foram encontrados 29 casos de hipospádia distal $(64,44 \%)$, seis casos de hipospádia média $(13,33 \%)$ e 10 casos de hipospádia proximal $(22,22 \%)$ (F-test $(2,8)=5,552$; $\left.P=0,003 ; X^{2}=9,3117 ; P=0,009\right)$. Houve predomínio da etnia branca, com registro de apenas um caso de hipospádia em pardo, não havendo relação na região entre a etnia e o tipo de hipospádia. Também não houve relação entre a etnia e a malformação associada aos tipos de hipospádia.

Do total de casos, $62 \%$ apresentavam algum tipo de malformação associada, e alguns dos casos, mais de uma malformação estava presente (Tabela 1).

Evidenciou-se que nos casos de hipospádia distal, as malformações mais simples como hérnias umbilicais foram encontradas em $6,8 \%$ ( 2 casos), hérnia inguinal 6,8\% ( 2 casos), hérnia inguinal bilateral $6,8 \%$ ( 2 casos), estenose de meato uretral $10,3 \%$ ( 3 casos), fimose $6,8 \%$ ( 2 casos), sendo que nesses casos não havia deformidade do prepúcio, apenas o meato uretral apresentou-se ventralizado ( $K W=3,322, \mathrm{P}=0,19)$.

Já em relação à hipospádia média, a malformação mais comum associada foi a hidrocele, com 33\% ( 2 casos). Em especial importância, o estudo evidenciou um caso de malformação cardíaca associado a esse tipo de hipospádia.

Contudo, na hipospádia proximal, encontrou-se um caso cuja malformação associada é uma síndrome genética que se caracteriza por presença de atraso de desenvolvimento neuropsicomotor, displasia de quadril além de fenda labial e dígito extranumerário. Este caso foi encaminhado para o Serviço de Genética Clínica da Faculdade de Ciências Médicas da Universidade Estadual de Campinas (SGM/ FCM/UNICAMP) e está sob investigação médica.

No HUSF constatou-se que o maior número de casos foi abordado de forma cirúrgica entre o terceiro e o quarto ano de idade ( $\mathrm{KW}=6,706$; $\mathrm{P}=0,022$ ) (Tabela 2). Houve dois casos de pacientes operados após o oitavo ano de vida. No tocante à técnica corretiva da hipospádia, observou-se que as técnicas de Mathiéu e MAGIP foram as mais utilizadas para correção das hipospádias distais. No caso das hipospádias proximais, foi utilizada a técnica de Duckett.
Tabela 1

Malformações associadas aos tipos de hipospádia.

\begin{tabular}{|c|c|c|c|}
\hline \multirow[t]{2}{*}{ Malformações Associadas } & \multicolumn{3}{|c|}{ Tipos de Hipospádia } \\
\hline & Proximal & Média & Distal \\
\hline Hérnia Inguinal & 1 & & 4 \\
\hline Hérnia Umbilical & 1 & 1 & 2 \\
\hline $\begin{array}{l}\text { Estenose de Meato } \\
\text { Uretral }\end{array}$ & 1 & 1 & 3 \\
\hline Criptorquidia & 1 & & 1 \\
\hline Hidrocele & & 2 & 1 \\
\hline Cisto de Prepúcio & & 1 & \\
\hline Ectopia Renal & & & 1 \\
\hline Fimose & 1 & & 2 \\
\hline Refluxo Vesicoureteral & & 1 & 1 \\
\hline $\begin{array}{l}\text { Malformação Cardíaca } \\
\text { Congênita }\end{array}$ & & 1 & \\
\hline Síndromes Genéticas & 1 & & \\
\hline $\begin{array}{l}\text { Atraso de desenvolvi- } \\
\text { mento Neuropsicomotor }\end{array}$ & 1 & & \\
\hline Dígito extranumerário & 1 & & \\
\hline Anquiloglossia & & & 1 \\
\hline Fenda Labial & 1 & & \\
\hline Displasia de quadril & 1 & & \\
\hline
\end{tabular}

\section{Tabela 2}

Número de abordagens cirúrgicas para correção dos diferentes tipos de hipospádia e idade à primeira cirurgia.

\begin{tabular}{ccccc}
\hline Idade à 1 ${ }^{\text {a }}$ cirurgia & \multicolumn{4}{c}{ Classificação da Hipospádia } \\
\cline { 2 - 5 } (emos) & Proximal & Média & Distal & Total \\
\hline $0-2.0$ & 0 & 0 & 1 & 1 \\
$3.0-4.0$ & 5 & 1 & 12 & 18 \\
$5.0-6.0$ & 3 & 1 & 7 & 11 \\
$7.0-8.0$ & 1 & 0 & 2 & 3 \\
Acima de 8 & 1 & 0 & 2 & 3 \\
Total & 10 & 2 & 24 & 36 \\
\hline
\end{tabular}

\section{Tabela 3}

Local de origem dos pacientes, número de casos e habitantes dos municípios da microrregião de Bragança Paulista. *Monte Verde/MG não faz parte da microrregião, porém apresentou um caso atendido no período entre 2007 e 2017.

\begin{tabular}{lcc}
\hline Cidade & Casos & População \\
\hline Bragança Paulista & 22 & 164.163 \\
Atibaia & 7 & 139.683 \\
Bom Jesus dos Perdões & 4 & 24.023 \\
Socorro & 2 & 37.288 \\
Nazaré Paulista & 1 & 18.121 \\
Pedra Bela & 1 & 6.154 \\
Joanópolis & 3 & 12.947 \\
Pinhalzinho & 2 & 13.104 \\
Tuiuti & 2 & 6.689 \\
Monte Verde/MG* & 1 & 4.131 \\
Total & 45 & 426.303 \\
\hline
\end{tabular}




\section{DISCUSSÃO}

O estudo epidemiológico da hipospádia teve seu início com Sørensen $(1953)^{12}$, realizado na Dinamarca entre 1910 e 1945, para elucidar as causas da malformação.

A hipospádia acomete de 4,4 a $8 \%$ dos meninos nascidos vivos nos EUA, é a malformação mais frequente da genitália externa masculina, dado que mostra a importância do conhecimento da doença para o médico generalista da atenção primária, para o pediatra e ao cirurgião pediátrico ${ }^{13}$. No Canadá, a incidência apresenta uma variação ainda maior - de 0,4 a 8,2 casos por 1000 meninos nascidos vivos ${ }^{14,15}$. Entretanto, a prevalência encontrada no presente trabalho foi próxima aos valores mais baixos para populações europeias $^{9}$. Tal fato pode ter sido influenciado pela predominância da etnia branca de origem europeia na população da Região Bragantina. Mesmo se apresentando mais comum em caucasianos, intermediária entre os afro-americanos e menos comum nos hispânicos nos EUA ${ }^{16}$, não se pode considerar a etnia como fator de risco no Brasil devido à miscigenação da sua população. Neste caso, deve existir parcimônia na interpretação da prevalência de hipospádia encontrada na região Bragantina, impossibilitando generalizações para a população brasileira. Desta forma, corroborando com Paulozzi et al. (1997) ${ }^{16}$, a variação da prevalência ocorre devido a diferenças geográficas, genéticas e ambientais, além de diferentes métodos para a coleta de dados.

Nos anos 1980 houve um aumento da incidência de casos na Europa e América do Norte. Esse crescimento se deveu talvez ao aumento do número de prematuros com baixo peso ou a exposição fetal a alimentos que contenham progesterona ou estrogênio ${ }^{17}$. Alguns autores sugerem que uma dieta rica em alimentos que contenham fitoestrogênio contribua para o aumento da incidência de hipospádia ${ }^{18,19}$.

No presente estudo, a maior parte dos pacientes apresentava hipospádia distal, seguida do tipo proximal e média. Os dados são semeIhantes aos encontrados por De Almeida Moreira $(2017)^{20}$, em estudo realizado no Serviço de Cirurgia Pediátrica da Universidade do Porto entre 2013 e 2016 , no qual relatou $83,3 \%$ de casos de hipospádia distal, 9,5\% de casos de hipospádia média e $7,1 \%$ de casos de hipospádia proximal e Kalfa et al. (2015) ${ }^{21}$, que encontraram na região de Montpellier, entre $2009-2014,70 \%$ de casos de hipospádia distal, $21,7 \%$ de casos de hipospádia média e $8,3 \%$ de casos de hipospádia proximal.

É de fundamental importância a pesquisa das anmalias associadas à hipospádia. O presente estudo evidenciou malformações simples como hérnia umbilical, hérnia inguinal, hérnia inguinal bilateral, estenose de meato uretral e fimose estão mais associados ao tipo distal de hipospádia. Martins et al. (2013) 22 encontraram $8 \%$ (4 pacientes) que apresentavam hérnia inguinal relacionada à hipospádia distal no Hospital Infantil Joana de Gusmão (Florianópolis/SC) entre 2011 e 2013. Entretanto, a literatura não justifica porque esses tipos de malformação estão mais relacionados com a modalidade distal de hipospádia. Segundo a literatura, a hérnia inguinal e o criptorquidismo são as anomalias mais comuns associadas com hipospádia4,23,24. Entre 8\% e $10 \%$ dos meninos com hipospádia tem criptorquidismo, e de $9 \%$ a $15 \%$ apresentam hérnia inguinal associada ${ }^{4}$. Pediatras e cirurgiões pediátricos precisam suspeitar da possibilidade de distúrbios do desenvolvimento sexual em crianças com hipospádia e criptorquidismo e que apresentem testículos não palpáveis. Destaca-se no presente estudo um caso de hipospádia distal com presença de anquiloglossia como malformação associada.

Em estudo sobre hipospádia proximal, realizado no Hospital Infantil da Faculdade de Medicina de Hannover entre os anos de 1980 a 1995 com 48 crianças e adolescentes entre 1 e 18 anos, Albert et al. (1997) ${ }^{14}$ encontraram os seguintes distúrbios do desenvolvimento sexual associados: Síndrome de Drash com tumor de Wilms, hermafroditismo verdadeiro, aberração cromossômica, deficiência do hormônio antimülleriano, disgenesia gonadal, deficiência parcial da enzima 5-alfa redutase causada por mutação, mutação no receptor de androgênio, malformação cardíaca, atresia retal e dilatação do trato urinário. A importância do diagnóstico destes distúrbios do desenvolvimento sexual está ligada ao risco aumentado de neoplasia maligna nas gônadas com alteração genética. Estima-se o aumento de $25 \%$ na probabilidade de neoplasia gonadal em pacientes com cariótipo $45 \mathrm{X}^{25}$. 
A literatura também relata que anomalias do trato urinário como obstrução da junção ureteropélvica, refluxo vesicoureteral, rim em ferradura, ectopia renal e agenesia renal ocorrem em $5 \%$ dos casos de hipospádia proximal ${ }^{4}$. A real incidência de hipospádia grave não é sabida, pois a diferenciação entre hipospádia proximal e genitália ambígua é difícil de ser realizada ${ }^{14}$. A presença de chordee, do latim chorda, refere-se à curvatura ventral do pênis. Ela é causada pela atrofia do corpo esponjoso e por fibrose da túnica albugínea. Tal característica está mais comumente associada a casos de hipospádia proximal ${ }^{26}$.

No presente estudo, constatou-se um caso de hipospádia em familiares (irmão). Bauer et al. (1979) 27 acompanharam 177 crianças com hipospádia por cinco anos no Hospital Infantil da Faculdade de Medicina de Harvard e observaram que em $25 \%$ dos casos havia outro membro da família com hipospádia, além de $12 \%$ com três membros da família afetados. Segundo esse estudo, $14 \%$ dos irmãos apresentavam também alguma modalidade de hipospádia, $9 \%$ dos pais, $3 \%$ dos primos e 2,2\% dos tios também apresentavam a anormalidade. Já Leung et al. (1985) ${ }^{28}$, em uma série de 1314 casos de hipospádia, observaram que em $5,4 \%$ dos casos outro membro da família também apresentava a anomalia. $O$ aumento da incidência familiar de hipospádia se deve provavelmente a uma predisposição poligênica ${ }^{15}$. Em geral, o risco de um segundo membro da família apresentar hipospádia aumenta com a gravidade do caso inicial ${ }^{29}$. A herança de um gene dominante talvez seja responsável por um número pequeno de casos entre familiares. Page $(1979)^{30}$ relatou seis casos de hipospádia em três ou mais gerações em uma mesma família, sugerindo uma herança dominante mendeliana.

A maior parte dos casos de hipospádia do HUSF foi abordada entre os três e os quatro anos de idade do paciente. Contudo, a idade ideal para o reparo cirúrgico em uma criança saudável é de aproximadamente seis a 12 meses de idade ${ }^{31,32}$. As vantagens para a cirurgia precoce incluem fácil pós-operatório devido a menor necessidade de analgesia, menor tempo de cateterismo urinário, maior facilidade de higienização, menos distúrbios emocionais no período pós-cirúrgico ${ }^{33}$. Mureau et al. $(1995)^{34}$ constataram que garotos que tiveram correção cirúrgica precoce de hipospádia apresentaram menos inibição sexual comparado com garotos que foram operados com maior idade.

A conduta cirúrgica na hipospádia visa correção estética e funcional da genitália masculina. O prepúcio exuberante que cobre apenas a face dorsal da glande deve ser corrigido para compensar a falha ventral. Do ponto de vista funcional, a topografia ectópica do meato uretral implica num jato urinário não-direcionável para frente. A principal consequência desse fato é que as crianças são obrigadas a urinar sentadas, sendo compreensível o problema psicológico que isso acarreta. A presença de tecido fibroso na superfície do pênis e a consequente curvatura peniana ventral congênita representa outro aspecto funcional a ser corrigido ${ }^{35}$. No HUSF, o presente estudo constatou que a principal técnica cirúrgica utilizada foi a de Mathiéu, esta utilizada para correção de hipospádia distal. Isto ocorre porque essa modalidade de hipospádia apresenta maior incidência de casos no serviço.

\section{CONCLUSÃO}

Conclui-se que a prevalência de hipospádia na população Bragantina é baixa e que o tipo distal de hipospádia foi o mais comum no HUSF no período entre 2007 e 2017, englobando a maior parte das malformações associadas. Observou-se, ainda, que a idade à primeira cirurgia estava em desacordo com o intervalo proposto para a abordagem corretiva da hipospádia.

\section{REFERÊNCIAS}

1. Maksoud JG. Cirurgia pediátrica. Rio de Janeiro: Revinter; 1998.

2. Sweet RA, Schrott HG, Kurland R, Culp OS. A study of the incidence of hypospadias in Rochester Minnesota 1940-1970 and a case-control comparison of possible etiologic factors. Mayo Clin Proc. 1974;49(1):52-58.

3. Streit D. Hipospadia. In: Bedhack DA, Damião R (editores). Guia prático de urologia. São Paulo: BG Cultural; p. 351-56, 1999.

4. Khuri FJ. Urological anomalies associated with hypospadias. Urol Clin North Am. 1981;8:565. 
5. Moreno-García M, Miranda EB. Chromosomal anomalies in cryptorchidism and hypospadias. J Urol. 2002; 168(5):2170-2.

6. Ikoma $\mathrm{F}$, Shima $\mathrm{H}$, Yabumoto $\mathrm{H}$. Classification of enlarged prostatic utricle in patients with hypospadias. $\mathrm{Br} \mathrm{J}$ Urol. 1985;57(3):334-7.

7. Duckett JW. Hypospadias. Campbell's Urology. Philadelphia: Saunders; 1998.

8. Baskin LS, Erol A, Li YW, Cunha GR. Anatomical studies of hypospadias. J Urol. 1998;160(3):1108-15.

9. Dolk H, Vrijheid M, Scott JE, Addor MC, Botting B, de Vigan $C$, et al. Toward the effective surveillance of hypospadias. Environ Health Perspect. 2004;112(3):398-402.

10. Gatti JM, Kirsch AJ, Troyer WA, Perez-Brayfield MR, Smith EA, Scherz HC. Increased incidence of hypospadias in small-for-gestational age infants in a neonatal intensive-care unit. BJU Int. 2001;87(6):548-50.

11. IBGE. Instituto Brasileiro de Geografia e Estatística. Dados demográficos da microrregião de Bragança Paulista. 2016.

12. Sørensen HR. Hypospadias. With special reference to ætiology. Transl. from the Danish by Anna la Cour. Munksgaard. Tese [Doutorado em Medicina]. 1953.

13. Belman AB. Hypospadias update. Urology. $1997 ; 49(2): 166-72$.

14. Albert N, Ulrichs $C$, Glüer $S$, Hiort $O$, Sinnecker $G H$, Mildenberger $\mathrm{H}$, et al. Etiologic classification of severe hypospadias: implications for prognosis and management. J Pediatr. 1997;131(3):386-92.

15. Gallentine ML, Morey AF, Thompson IM Jr. Hypospadias: a contemporary epidemiologic assessment. Urology. 2001;57(4):788-90.

16. Paulozzi LJ, Erickson JD, Jackson RJ. Hypospadias trends in two US surveillance systems. Pediatrics. 1997;100(5):831-4.

17. Chavez GF, Cordero JF, Becerra JE. Leading major congenital malformations among minority groups in the United States, 1981-1986. MMWR Morb Mortal Wkly Rep. 1988;37:17-24.

18. North K, Golding J, ALSPAC Study team. A maternal vegetarian diet in pregnancy is associated with hypospadias. BJU Int. 2000;85(1):107-13.

19. Joffe M. Are problems with male reproductive health caused by endocrine disruption? Occup Environ Med. $2001 ; 58(4): 281$.
20. De Almeida Moreira C. Caracterização dos casos de hipospadia em crianças nascidas entre 2013 e 2016 no CMIN/CHP. Porto. Dissertação [Mestrado integrado em Medicina]. Universidade do Porto; 2017.

21. Kalfa N, Paris F, Philibert $P$, Orsini M, Broussous $S$, Fauconnet-Servant $\mathrm{N}$, et al. Is hypospadias associated with prenatal exposure to endocrine disruptors? A French collaborative controlled study of a cohort of 300 consecutive children without genetic defect. Eur Urol. 2015;68(6):1023-30.

22. Martins AV, Araujo EJD, Souza JAD, Colombeli EM, Lima RM, Ramos TC. Tratamento cirúrgico da hipospadia distal. Arq Catarin Med. 2013;42(4):54-9.

23. Weidner IS, Moller H, Jensen TK, Skakkebaek NE. Risk factors for cryptorchidism and hypospadias. J Urol. 1999; 161(5):1606-9.

24. Leung AK, Robson WL. Current status of cryptorchidism. Adv Pediatr. 2004; 51:351-77.

25. Aarskog D. Clinical and cytogenetic studies in hypospadias. Acta Paediatr Scand Suppl. 1970;203(Supl.203).

26. Soomro NA, Neal DE. Treatment of hypospadias: an update of current practice. Hosp Med. 1998; 59(7):553-556.

27. Bauer SB, Bull MJ, Retik AB. Hypospadias: a familial study. J Urol. $1979 ; 121(4): 474-7$.

28. Leung TJ, Baird PA, McGillivray B. Hypospadias in British Columbia. Am J Med Genet. 1985;21(1):39-48.

29. Kenneth $\mathrm{CH}$, Leung AK. Hypospadias: a review. J Singapore Paediatr Soc. 1987;29:54-6.

30. Page LA. Inheritance of uncomplicated hypospadias. Pediatrics. 1979;63(5):788-90.

31. American Academy of Pediatrics. Timing of elective surgery on the genitalia of male children with particular reference to the risks, benefits and psychological effects of surgery and anesthesia. Pediatrics. 1996;97:590-4.

32. Manzoni G, Bracka A, Palminteri E, Marrocco G. Hypospadias surgery: when, what and by whom? BJU Int. 2004;94(8):1188-95.

33. Leung AK. Hypospadias. Core Content Rev Fam Med. 2004;35:17.

34. Mureau MA, Slijper FM, van der Meulen JC, Verhulst FC, Slob KA. Psychosexual adjustment of men who underwent hypospadias repair: norm-related study. J Urol. $1995 ; 154(4): 1351-5$.

35. Macedo AJR, Srougi M. Hipospadias. Rev Assoc Med Bras. $1998 ; 44(2): 141-5$. 